indicates a high relevance of the questionnaire items to the subject. At this stage, modifications have been made to eliminate ambiguity and repetition. We will now test concurrent validity, specificity and test-retest variability of the questionnaire in healthy volunteers, and respiratory patients with and without VCD.

\section{P228 AN AUDIT OF THE EFFECTIVENESS OF COMPETENCY BASED SPIROMETRY TRAINING}

doi:10.1136/thoraxjnl-2011-201054c.228

${ }^{1} \mathrm{~J}$ Shakespeare, ${ }^{2} \mathrm{~K}$ Storey, ${ }^{1} \mathrm{D}$ Parr. ${ }^{1}$ University Hospitals Coventry and Warwickshire, Coventry, England; ${ }^{2}$ Coventry Primary Care Trust, Coventry, England

Introduction The PCT had funded practice nurses to undertake formal competency based training in spirometry measurements. An audit of spirometry provision in practices within the PCT was undertaken to assess equipment, training and quality of spirometry being performed with comparison made between spirometry undertaken by trained staff (ARTP Cert) and that performed by untrained staff. The aim was to assess the effectiveness of the competency based training being funded by the PCT.

Methods 36 practice nurses had received training from Respiratory Physiologists based in an acute hospital trust to ARTP Full Certificate in Spirometry standards. 62 Primary Care practices were sent a questionnaire, designed by the author, and asked to supply five recent anonymous spirometry traces.

Results 26 practices responded (42\%); 5 (19\%) did not perform spirometry testing, due to a "lack of staff skills" (4/5) and "young patient population" $(1 / 5)$. Of those practices performing spirometry, all were using the Care Fusion MicroLab spirometer and the following training had been undertaken; ARTP training course (16/ $21)$, drug representative training (3/21), COPD Diploma (1/21) and no response $(1 / 21) .81 \%$ of practices performing spirometry had a calibration syringe and performed calibration either at each session or weekly. Training for the practices that did not have a calibration syringe (19\%) was by; COPD Diploma (1/4) and drug representative (3/4). 18/21 practices performing spirometry sent five traces for analysis. Only $17 \%$ practices performed relaxed VC manoeuvres and of these $66 \%$ achieved acceptability criteria. 13/18 performed the recommended minimum of three FVC's (72\%) with 11 of these (85\%) achieving two results within $5 \%$ or $100 \mathrm{ml}^{1}{ }^{5} 5 / 18$ did not perform a minimum of three manoeuvres (for three practices only one of the five traces met acceptability criteria, two of whom were ARTP spirometry trained). Two practices submitted five traces where none of the traces achieved the required acceptability criteria (both Drug Representative trained).

Conclusion Training staff to ARTP standards improves the quality of spirometry performed in primary care (when compared to other modes of training), however once training is completed, it is important to audit quality standards to ensure that they are still met.

\section{REFERENCE}

1. ARTP/BTS Guidelines Respiratory Medicine. 1994.

\section{P229 FACTORS AFFECTING INHALER CHOICE AND ADHERENCE IN URBAN LIVERPOOL}

doi:10.1136/thoraxjnl-2011-201054c.229

G Tack, E Tjia-Leong, L Davies, C J Warburton. University Hospital Aintree, Liverpool, UK

Introduction Salmeterol/Fluticasone propionate (SFC) in the form of a dry powder inhaler (DPI) is often used in COPD, however it is also available as a metered dose inhaler (MDI). This is unlicensed for
COPD and costs considerably more. At our hospital, a high proportion of COPD patients used SFC MDI, the reasons for this were unclear. This study aimed to investigate the effects of patient preference on inhaler adherence.

Methods Patients admitted to hospital with an exacerbation of COPD taking either SFC DPI or MDI were recruited. All patients completed a pre-discharge questionnaire about their inhaler usage. MDI patients were switched to DPI, following education and check of their inspiratory flow. GPs were informed that their patient was involved in a study of inhalers but not the detail of the study. All patients underwent a further questionnaire at 3 months.

Results 101 patients, mean (SD) age was 69 (9) years and 50\% male. On admission, 66 (65\%) on MDI, 35 (35\%) on DPI. $100 \%$ of MDI patients were switched to DPI. At 3 months, follow-up data were available on 81 patients. Of those patients admitted on DPI, 26/29 $(92 \%)$ remained on it and were satisfied with it. In the group switched from MDI to DPI, 26/52 (50\%) were again receiving MDI at 3 months. 16 patients had asked for their prescription to be changed back. 10 patients had their prescription changed without their knowledge or did not receive DPI on discharge. Regardless of the reasons for the switch, 18 patients stated they preferred MDI over DPI. Reasons why patients requested the change back to MDI included dry powder irritating the throat, dry mouth and the inhaled dose not going into the lungs.

Conclusion Following a relatively simple intervention $50 \%$ of COPD patients using SFC MDI could be switched and maintained on SFC DPI. Factors relating to a return to MDI included patient related and organisational factors. A whole system approach is required to effect robust systematic change in this patient group, however approximately a third of the group switched to DPI will still request a change back to MDI.

\section{P230 DO WE NEED A "TWO WEeK RULE" REFERRAL PATHWAY FOR LUNG CANCER?}

doi:10.1136/thoraxjnl-2011-201054c.230

M H Ali, A Berry, J Van Griethuysen, H Peters, A Jameel, G Haji, F Shora, M P Berry, E F Bowen. Imperial College NHS Trust, London, UK

Introduction The introduction of the two week rule (TWR) cancer referral system aimed to expedite the diagnosis and treatment of patients with lung cancer. There have been concerns that the system may even lead to delays and there remains little evidence to support its use. We therefore used prospective data to assess the effectiveness of this system.

Methods We prospectively collected data on patients referred in 2010 with suspected lung cancer to a large West London lung cancer centre. We evaluated final diagnosis, performance status, lung cancer staging, time until seen in specialist clinic and time until first treatment for patients referred under the TWR compared with patients referred via our in-house abnormal radiology referral service. Results In total 249 patients were included in the study (181 from radiology reporting and 68 from GP referrals via the 2 week wait). 83 (33\%) cancers were diagnosed from a total of 249 referrals. Patients referred from the radiology department were significantly more likely to have a diagnosis of lung cancer $(73 / 181,40 \%)$ than patients referred under the TWR $(10 / 68,15 \% ; p<0.001)$. The mean time from date of referral to seeing a specialist was similar in both groups. All patients diagnosed with lung cancer referred through the TWR had an abnormal chest radiograph. More patients with a performance status $0-1$ and earlier stage disease were referred from radiology than through the TWR

Conclusions A robust radiology referral system is an effective alternative method to diagnosing lung cancer than the TWR. Patients referred from radiology are significantly more likely to have lung cancer. We propose that out-patient clinic slots are reserved for 